\title{
FULLY AUTOMATIC SEGMENTATION OF THE DENTATE NUCLEUS USING DIFFUSION WEIGHTED IMAGES
}

\author{
Chuyang Ye $e^{1}$, John A. Bogovic ${ }^{1}$, Pierre-Louis Bazin ${ }^{2}$, Jerry L. Prince ${ }^{1}$ and Sarah H. Ying ${ }^{3}$ \\ ${ }^{1}$ Department of Electrical and Computer Engineering, Johns Hopkins University, \\ Baltimore, MD, USA \\ ${ }^{2}$ Department of Neurophysics, Max Planck Institute for Human Cognitive and Brain Sciences, \\ Leipzig, Germany \\ ${ }^{3}$ Departments of Radiology, Neurology, and Ophthalmology, Johns Hopkins University School of Medicine, \\ Baltimore, MD, USA
}

\begin{abstract}
We propose a fully automatic method to segment the dentate nucleus using diffusion weighted images (DWI). Initialization of the dentate nucleus is produced by combining the information from tractography results on the diffusion tensor images (reconstructed from DWI) and b0 images. A geometric deformable model (GDM) with generalized gradient vector flow (GGVF) is then applied on the b0 image to generate the segmentation by evolving the level set function. Experiments have been carried out on real data and quantitative comparison shows that our segmentation results agree well with expert manual delineations and produce accurate results.
\end{abstract}

Index Terms - segmentation, dentate nucleus, diffusion weighted images, geometric deformable model, generalized gradient vector flow

\section{INTRODUCTION}

The dentate nucleus is a structure located in the cerebellum that plays a significant role in the communications between the cerebellum and the rest of the brain [1]. It receives afferent projections not only from the cerebellar cortex but from outside the cerebellum as well, such as the spinal cord. It also outputs signals to other components of the nervous system [2]. The dentate nucleus is responsible for functions such as planning, initiation and control of volitional movements [3]. Numerous studies on its structure [4], function [5] and diseases related to the dentate nucleus [6] have been carried out. A number of studies [7, 6, 8] have demonstrated that magnetic resonance images (MRI) provide enough contrast to perform manual segmentation. However, manual delineations are subject to variability and are time-consuming. An automatic method to segment the dentate nucleus could dramatically advance research.

This work is partially sponsored by NIH/NINDS 5R01NS056307 and the China Scholarship Council

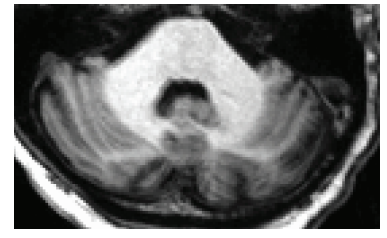

(a)

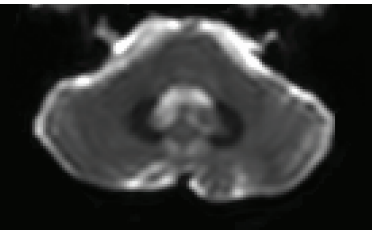

(b)
Fig. 1. Axial view of (a) MP-RAGE and (b) b0 image in the same slice. Images are zoomed into the cerebellum.

To the best of our knowledge, there exist no automatic methods to segment the dentate nucleus. Although with an atlas [8], segmentation is possible using the segmentationby-registration approach, more refined segmentations for precise volume quantification or for alternative atlas generation need more accurate and fully automatic methods. In this paper, we propose a fully automatic method for segmenting the structure using diffusion weighted images (DWI). The contrast of the dentate nucleus on MRI is usually low or even non-existing. For instance, as is shown in Figure 1(a) on an MP-RAGE image, the dentate nucleus is not visible. The dentate nucleus is visible on T2 weighted images, but contrast is typically poor. Similar contrast is found on b0 images in DWI where no gradient directions are applied, which is shown in Figure 1(b). Low level methods such as K-means or fuzzy Cmeans (FCM) clustering and region growing are difficult on $\mathrm{T} 2$ or b0 images for two main reasons. Firstly, it is necessary to begin with anatomical information. Simply using clustering will end up with too many structures that have similar intensities as the dentate nucleus. Edge information is also ignored in clustering. Secondly, because of this low contrast, methods such as region growing can easily extend out of the dentate nucleus and a threshold is hard to determine. Thus, we make use of anatomical connectivity information provided by tractography for initialization and apply a geometric 
deformable model (GDM) which includes both edge and intensity information to segment the dentate nucleus. Since we will use DWI to reconstruct the diffusion tensor images (DTI) to perform tractography and b0 images are part of DWI, the GDM is applied on b0 images. An example of our segmentation result is shown in Figure 2(a). Based on the fact that

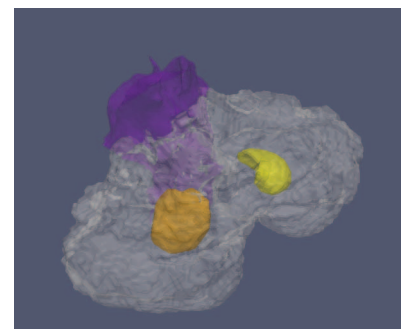

(a)

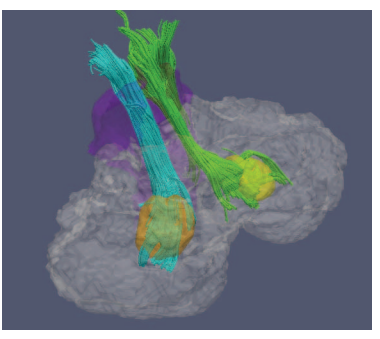

(b)
Fig. 2. An oblique view of the segmentation of the dentate nucleus and the superior cerebellar peduncles (SCP) shown together with the brainstem (purple) and the cerebellum (transparent). Orange/yellow volume: left/right dentate nucleus; blue/green fibers: left/right SCP. (a) Without SCP. (b) With SCP.

the superior cerebellar peduncles (SCP) originate from the dentate nucleus [3], we use the diffusion oriented tract segmentation (DOTS) algorithm [9] together with tractography to automatically obtain the SCP fibers. With the help of the SCP, it is possible to get an initialization of the dentate nucleus on b0 images. Then we apply a GDM combined with generalized gradient vector flow (GGVF) [10] on b0 images to accomplish the segmentation. The experiments on real data demonstrate that the method achieves accurate segmentation of the dentate nucleus.

\section{METHODS}

\subsection{Labeling of the SCP Fibers}

DOTS [9] is an atlas-based algorithm for white matter tract segmentation that directly labels each voxel within a Markov random field (MRF) framework. Computed fibers can also be automatically labeled by tract given the underlying DOTS voxel labels [9]. Here we first reconstruct tensors from DWI using CATNAP [11] and then apply DOTS on the DTI. Fibers from FACT [12] are labeled using DOTS results [9]. An example of the labeled SCP fibers is displayed in Figure 2(b). Note that the left SCP and right SCP are labeled separately. In this example, we can see that the method produces a reasonable labeling of the SCP fibers and that most SCP fibers originate from the dentate nucleus.

\subsection{Initialization of the Dentate Nucleus}

To obtain an initialization of the dentate nucleus, we combine the information from the SCP fibers and b0 images as the SCP originate from the dentate nucleus [3]. We extract the end points of the SCP, and then separate the end points of the SCP fibers into two groups and select the one nearest to the dentate nucleus. This is done by choosing the group that has more points inside the cerebellum mask generated by TOADS, a whole brain segmentation algorithm [13], on an MP-RAGE image of the same subject, because the other side is expected extend out of the cerebellum. Because of noise and the choice of parameters in tractography, some end points may not end in or near the dentate nucleus. Therefore we limit our selection to the end points in the center of the point cloud. For each point, the sum of the Euclidean distances to all others is calculated. Then we sort the points in ascending order by the sum and choose the first $10 \%$. This process is performed for left and right SCP separately.

B0 images provide sufficient contrast to distinguish the dentate nucleus from the neighboring voxels. A two-class FCM [14] is applied on the b0 image masked to include only the cerebellum. It can be observed in Figure 3(a) that the hard segmentation result includes parts of the dentate nucleus together with structures far from it. Therefore, we first partition the hard segmentation into isolated components with 26-connectedness. Then we calculate the sum of the distance from the end points extracted above to each component and select the one with the smallest sum of distance to be the initialization. Since we label left and right SCP separately, the initialization of the dentate nucleus can discriminate left and right side, as shown in Figure 3(b).

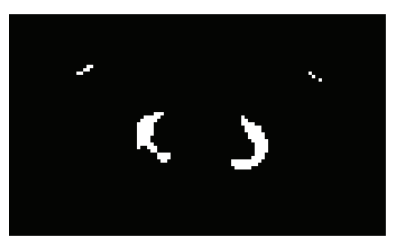

(a)

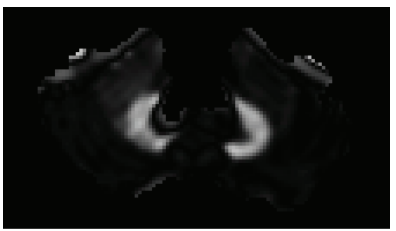

(c)

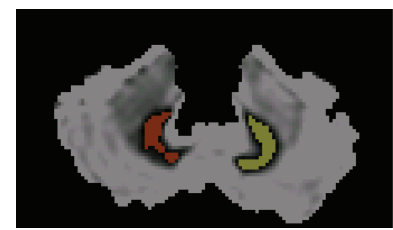

(b)

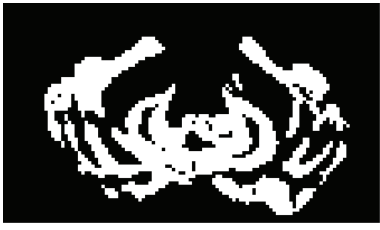

(d)
Fig. 3. An axial view of intermediate results. (a) FCM hard segmentation. (b) Final initialization (orange/yellow: left/right dentate nucleus) overlaid on contrast enhanced b0 image. (c) FCM membership. (d) Edge map. 


\subsection{Segmentation Using GDM}

With the initialization described above, a more accurate dentate boundary is found by using a GDM. The partial differential equation for the GDM level set is

$$
\phi_{t}+F_{0}|\nabla \phi|+F_{e x t} \cdot \nabla \phi=\epsilon \kappa|\nabla \phi|
$$

where $\phi$ is the level set function, $\kappa$ is the curvature, $F_{0}$ is the region force and $F_{\text {ext }}$ is the external force [15]. The region force assures that the level set expands inside the dentate nucleus and shrinks outside it. The region force is defined by membership obtained in the FCM result. Figure 3(c) gives an example of the membership. An empirical threshold $\tau(<0.5)$ is set such that the region force is positive where the membership is above $\tau$ and negative otherwise (Equation 2).

$$
F_{0}(x)= \begin{cases}\alpha, \quad M(x)>\tau \\ -\alpha, & \text { otherwise }\end{cases}
$$

Here $\alpha$ is the strength of the force, $x$ is the coordinate of the voxel and $M(x)$ is the membership at voxel $x$. We set $F_{\text {ext }}$ as the GGVF force. The GDM using GGVF has a large capture range and can converge into boundaries with concavities [16]. It also gives good results with the existence of weak edges [16]. To obtain the GGVF, an edge map is produced by applying 3-D Sobel operator on the Gaussian smoothed b0 image and then thresholding the result to get a binary map. An example is shown in Figure 3(d). Then the GGVF is calculated with the edge map by solving Equation 3 [10].

$$
F_{\text {ext }}=\underset{f}{\arg \min } \int_{\Omega} g(|\nabla f|)|\nabla \vec{v}|^{2}+h(|\nabla f|)|\vec{v}-\nabla f|^{2} d \omega
$$

where $g(|\nabla f|)=e^{-|\nabla f| / K}, h(|\nabla f|)=1-g(|\nabla f|)$, and $\vec{v}$ is the gradient field of the edge map. Note that the although the edges in the map are thick due to the low contrast between the dentate nucleus and its surroundings, with our definition of the region force and the 'medial' property of GGVF, the zero level set will not stop at the inner boundary. Instead it will stop between the inner and outer boundary as a result of the interaction of $F_{\text {ext }}$ and $F_{0}$. With the forces defined above, the segmentation of the dentate nucleus is achieved by evolving the level set function using Equation 1.

\section{EXPERIMENTS}

Experiments are carried out on real data. The diffusion weighted images were acquired using a multi-slice, single shot EPI sequence. Each sequence had one b0 image and used 32 gradient directions. The images were obtained on a 3T MR scanner (Intera, Philips Medical Systems, Netherlands). The DWI were then co-registered to corresponding MP-RAGE images resampled to $1 \mathrm{~mm}$ isotropic resolution.

Segmentation of the dentate nucleus is performed as we described above. Experiments are carried out on 4 subjects.

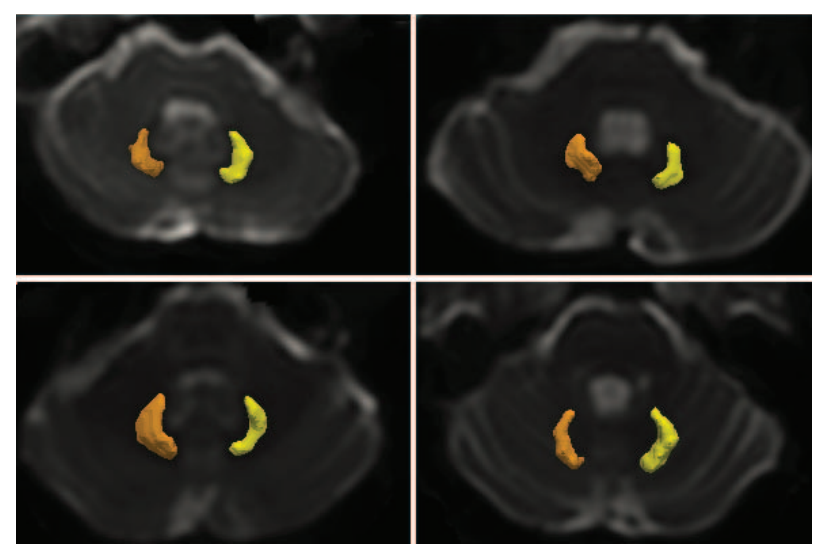

Fig. 4. Axial views of 3-D renderings of the segmentation result overlaid on b0 images on 4 subjects. B0 images are cropped to show the cerebellum. Orange/yellow volume: left/right dentate nucleus.

The results are shown in Figure 4 by overlaying the 3-D renderings of the segmentation on intersecting b0 images in an axial view. Axial and coronal cross sections are shown in Figure 5 for better appreciation of the details. Here the b0 image is displayed in a window and level chosen to better display the contrast between the dentate nucleus and its surroundings.

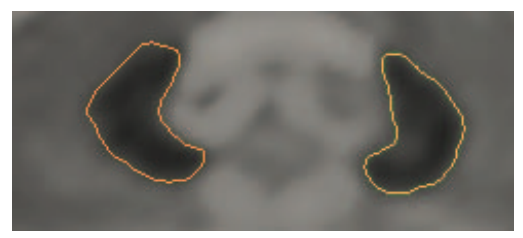

(a)

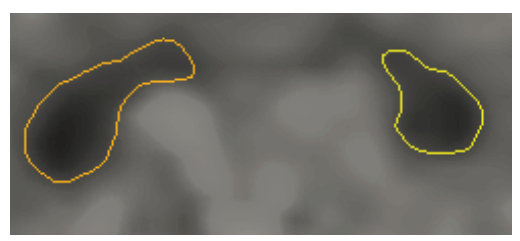

(b)

Fig. 5. Detail views of the contour of the segmentation for one subject overlaid on the contrast enhanced b0 image. Orange/yellow: left/right dentate nucleus. (a) Axial view. (b) Coronal view.

We have also compared our segmentation with manual delineations of the dentate nucleus. The left and right dentate nucleus are combined for comparison. The measurements are displayed in Table 1. In our segmentation result, the volume of the dentate nucleus is approximately $1 \mathrm{~cm}^{3}$. Given the small volume, the Dice coefficient and average surface distances indicate very good agreement between our automatic 
Table 1. Comparison between automatic segmentation and manual delineations

\begin{tabular}{|c|cccc|}
\hline Subject & 1 & 2 & 3 & 4 \\
\hline Dice coefficient & 0.88 & 0.89 & 0.88 & 0.86 \\
Average surface distance $(\mathrm{mm})$ & 0.31 & 0.42 & 0.37 & 0.36 \\
\hline
\end{tabular}

segmentation and manual delineations and show that the proposed method segments the dentate nucleus accurately.

\section{CONCLUSION}

In this paper, we presented a fully automatic method to segment the dentate nucleus using DWI. Diffusion weighted images are used in two ways. First, we combine diffusion information with high-level anatomical connectivity models in order to locate the dentate nucleus. Then this initialization allows us to segment the dentate nucleus directly on the b0 maps with a robust level set approach. Experiments show that despite the small size of the structure, the method produces results that agree well with manual labeling. Future work may include validation of the method on a larger cohort and application to the studies of the involvement of the dentate nucleus in neurodegenerative diseases.

\section{REFERENCES}

[1] F. Sultan, S. Hamodeh, and J.S. Baizer, "The human dentate nucleus: a complex shape untangled," Neuroscience, vol. 167, no. 4, pp. 965 - 968, 2010.

[2] C.Y. Saab and W.D. Willis, "The cerebellum: organization, functions and its role in nociception," Brain Research Reviews, vol. 42, no. 1, pp. 85 - 95, 2003.

[3] D. Ristanović, N.T. Milošević, B.D. Stefanović, D.L. Marić, and K.Rajkovič, "Morphology and classification of large neurons in the adult human dentate nucleus: A qualitative and quantitative analysis of 2D images," Neuroscience Research, vol. 67, no. 1, pp. 1-7, 2010.

[4] K. Yamaguchi and N. Goto, "Three-dimensional structure of the human cerebellar dentate nucleus: a computerized reconstruction study," Anatomy and Embryology, vol. 196, pp. 343-348, 1997.

[5] W.T. Thach, "Timing of activity in cerebellar dentate nucleus and cerebral motor cortex during prompt volitional movement," Brain Research, vol. 88, no. 2, pp. $233-241,1975$.

[6] C.W. Tjoa, R.H.B. Benedict, B. Weinstock-Guttman, A.J. Fabiano, and R. Bakshi, "MRI T2 hypointensity of the dentate nucleus is related to ambulatory impairment in multiple sclerosis," Journal of the Neurological Sciences, vol. 234, no. 1-2, pp. 17 - 24, 2005.

[7] A. Dimitrova, D. Zeljko, F. Schwarze, M. Maschke, M. Gerwig, M. Frings, A. Beck, V. Aurich, M. Forsting, and D. Timmann, "Probabilistic 3D MRI atlas of the human cerebellar dentate/interposed nuclei," NeuroImage, vol. 30, no. 1 , pp. $12-25,2006$.

[8] J. Diedrichsen, S. Maderwald, M. Kper, M. Thrling, K. Rabe, E.R. Gizewski, M.E. Ladd, and D. Timman$\mathrm{n}$, "Imaging the deep cerebellar nuclei: A probabilistic atlas and normalization procedure," NeuroImage, vol. 54, no. 3, pp. 1786 - 1794, 2011.

[9] P.-L. Bazin, C. Ye, J.A. Bogovic, N. Shiee, D.S. Reich, J.L. Prince, and D.L. Pham, "Direct segmentation of the major white matter tracts in diffusion tensor images," NeuroImage, vol. 58, no. 2, pp. 458 - 468, 2011.

[10] C. Xu and J.L. Prince, "Generalized gradient vector flow external forces for active contours," Signal Processing, vol. 71, no. 2, pp. $131-139,1998$.

[11] B.A. Landman, J.A.D. Farrell, N.-L. Patel, S. Mori, and J.L. Prince, "DTI fiber tracking: the importance of adjusting DTI gradient tables for motion correction. CATNAP - a tool to simplify and accelerate DTI analysis," in Proc. Org Human Brain Mapping 13th Annual Meeting. 2007.

[12] S. Pajevic, A. Aldroubi, and P.J. Basser, "A continuous tensor field approximation of discrete DT-MRI data for extracting microstructural and architectural features of tissue," Journal of Magnetic Resonance, vol. 154, no. 1, pp. $85-100,2002$.

[13] P.-L. Bazin and D.L. Pham, "Homeomorphic brain image segmentation with topological and statistical atlases," Medical Image Analysis, vol. 12, no. 5, pp. 616 $-625,2008$.

[14] J.C. Bezdek, R. Ehrlich, and W. Full, "FCM: The fuzzy c-means clustering algorithm," Computers \& Geosciences, vol. 10, no. 2-3, pp. 191 - 203, 1984.

[15] J.A. Sethian, Level Set Methods and Fast Marching Methods: Evolving Interfaces in Computational Geometry, Fluid Mechanics, Computer Vision, and Materials Science, Cambridge University Press, 2nd edition, June 1999.

[16] C. Xu, A. Yezzi Jr, and J.L. Prince, "A summary of geometric level-set analogues for a general class of parametric active contour and surface models," in Variational and Level Set Methods in Computer Vision, 2001. Proceedings. IEEE Workshop on, 2001, pp. $104-111$. 